\title{
Leptin induces the apoptosis of chondrocytes in an in vitro model of osteoarthritis via the JAK2-STAT3 signaling pathway
}

\author{
ZI MING ZHANG ${ }^{1}$, CHAO SHEN $^{2}$, HAI LI $^{1}$, QING FAN $^{1}$, JING DING $^{1}$, FANG CHUN JIN $^{1}$ and LIN SHA ${ }^{1}$ \\ Departments of ${ }^{1}$ Pediatric Orthopedics and ${ }^{2}$ Orthopedics, Xin Hua Hospital Affiliated to Shanghai Jiaotong University, \\ School of Medicine, Shanghai 200092, P.R. China
}

Received February 26, 2015; Accepted December 15, 2015

DOI: $10.3892 / \mathrm{mmr} .2016 .4970$

\begin{abstract}
Emerging data has suggested a high prevalence of osteoarthritis (OA) among obese people. As an important adipokine secreted by white adipose tissue, leptin may be a key mediator in the progression of OA. Leptin exerts a catabolic effect on OA cartilage by increasing the production of metalloproteinase (MMP) enzymes, and contributes to apoptosis in chondrocytes. The current study aimed to explore the role of leptin on the apoptosis of chondrocytes in OA, and its underlying mechanisms. In the in vitro model of OA used in the present study, administration of exogenous leptin induced the generation of reactive oxygen species (ROS) and apoptosis in chondrocytes. It has been demonstrated that leptin is associated with the pathogenesis of OA via the Janus kinase 2 (JAK2)-signal transducer and activator of transcription 3 (STAT3) signaling pathway, and data gathered in the present study demonstrated that suppression of this signaling pathway using a JAK2 inhibitor, AG490, significantly ameliorated leptin-induced apoptosis in damaged chondrocytes in vitro, and reduced the generation of ROS. Furthermore, the protein expression levels of MMP-13 and B-cell lymphoma 2-associated $X$ protein were downregulated in the AG490-treated group. The results of the present study may provide insight into the underlying molecular mechanism by which leptin induces apoptosis in chondrocytes. These findings indicated the importance of leptin as a therapeutic target for the treatment of $\mathrm{OA}$ in the overweight population.
\end{abstract}

\section{Introduction}

Osteoarthritis (OA) is a painful joint disease that develops due to insufficient and aberrant repair of damaged synovial joint

Correspondence to: Dr Zi Ming Zhang, Department of Pediatric Orthopedics, Xin Hua Hospital Affiliated to Shanghai Jiaotong University, School of Medicine, 1665 Kongjiang Road, Yangpu, Shanghai 200092, P.R. China

E-mail: zhangzm106@163.com

Key words: osteoarthritis, chondrocytes, leptin, apoptosis, JAK-STAT pathway tissue (1). The articular cartilage is altered to a certain extent in response to aging, inappropriate mechanical stress and low-grade inflammation associated with obesity (2). Obesity has long been recognized as a risk factor for the development of OA. Excess body weight may lead to cartilage degeneration and dysfunction of chondrocytes, due to increased mechanical forces. However, a strong association between obesity and OA in non-weight-bearing joints, including those in the fingers and wrists, has also been demonstrated (3). A previous study indicated that adipokines released by white adipose tissue (WAT) may be associated with the high prevalence of OA among obese people (4).

Leptin centrally regulates body weight and eating behavior, and is the most researched adipokine secreted by WAT (5). In addition, the role of leptin as a proinflammatory regulator in OA and the expression of leptin receptors in chondrocytes have previously been reported (6). During the development of OA, chondrocytes undergo gradual dedifferentiation, apoptosis is triggered by low-grade inflammation (7), and reactive oxygen species (ROS) are generated (8). Leptin has been demonstrated to enhance the expression of inducible nitric oxide synthase (iNOS) (9) and cartilage-degrading matrix metalloproteinases (MMP), including MMP-9 and MMP-13, which are involved in OA cartilage damage (10). This requires the activation of the Janus kinase 2 (JAK2)-signal transducer and activator of transcription 3 (STAT3), phosphatidylinositol-4,5-bisphosphate 3-kinase (PI3K) and mitogen-activated protein kinase (MAPK) signaling pathways $(9,11)$.

The underlying mechanism by which leptin induces chondrocyte apoptosis remains to be elucidated. In the present study, the role of the JAK2-STAT3 signaling pathway in leptin-induced chondrocyte apoptosis was investigated. To further understand the mechanism of action, characteristic autophagy markers were also investigated.

\section{Materials and methods}

Primary culture of chondrocytes from a model of $O A$. The present study was approved by the ethics committee of Xin Hua Hospital Affiliated to Shanghai Jiaotong University. A total of 12 male Sprague-Dawley rats (SD) rats (age, 10 weeks) were obtained from Shanghai Experimental Animal Center (Shanghai, China), and were housed at ambient temperature $\left(23^{\circ} \mathrm{C}\right)$ under a $12 \mathrm{~h} / 12 \mathrm{~h}$ light-dark cycle, with free access to 
chow and water. SD rats were anesthetized with $4 \%$ halothane in oxygen, and were subsequently shaved and disinfected with $70 \%$ ethanol. The right knee joint was exposed by the medial parapatellar approach. The patella was dislocated laterally and the knee placed in full flexion, followed by anterior cruciate ligament transection (ACLT) using micro-scissors, in order to induce ACLT-mediated knee OA, as previously described (12). In the control group, the right knee joint was exposed and incisions were sutured following subluxation of the patella and washing of the joint surface with saline. Following full post-operative recovery, rats were sacrificed by anesthesia (4\% halothane in oxygen) and cartilage samples from the knee joints of the OA model and control group rats were removed and washed several times with phosphate-buffered saline (PBS). Type II collagenase (Sigma-Aldrich, St. Louis, MO, USA) was used to digest the cartilage at $37^{\circ} \mathrm{C}$ for $5 \mathrm{~h}$, and the solution was centrifuged for $5 \mathrm{~min}$ at $200 \mathrm{x} \mathrm{g}, 4^{\circ} \mathrm{C}$. Primary chondrocytes were cultured in Dulbecco's modified Eagle's medium (DMEM) supplemented with $1 \%$ fetal bovine serum (Hyclone; GE Healthcare Life Sciences, Logan, UT, USA). Cells were seeded in a monolayer at $50-60 \%$ density for use in further studies.

Identification of chondrocytes. Freshly isolated chondrocytes from the control group were cultured to 50-60\% density and fixed with $4 \%$ paraformaldehyde at room temperature for $30 \mathrm{~min}$. Following several washes with PBS, the cells were treated with $3 \% \mathrm{H}_{2} \mathrm{O}_{2}$ and blocked with non-specific normal anti-goat serum (Beyotime Institute of Biotechnology, Shanghai, China). A previous study indicated that expression of collagen II was a marker for functional chondrocytes (13). The rabbit anti-rat polyclonal collagen II antibody (1:200; cat. no. ab34712; Abcam, Cambridge, MA, USA) was applied overnight at $4^{\circ} \mathrm{C}$, followed by incubation with horseradish peroxidase-conjugated goat anti-rabbit IgG secondary antibody (1:1,000; cat. no. ab6721; Abcam) at $37^{\circ} \mathrm{C}$ for $20 \mathrm{~min}$. Cells incubated with immunoglobulin ( $\left.\mathrm{Ig}\right) \mathrm{G}$ (1:200; cat. no. A7016; Beyotime Institute of Biotechnology) were used as negative controls for the immunocytochemistry. 3,3'-Diaminobenzidine staining was used to indicate the presence of collagen II and images were captured using an Eclipse Ti-S microscope (Nikon Corporation, Tokyo, Japan) at a magnification of $\mathrm{x} 200$. Image J version 1.49 software (imagej.nih.gov/ij) was used for quantitative analysis of positive areas.

Cell viability assay. Chondrocytes $\left(3 \times 10^{3}\right.$ per well) were plated in 96-well plates. A series of leptin concentrations $(2.5,5,10$, 20 or $50 \mathrm{ng} / \mathrm{ml}$; Sigma-Aldrich) or $20 \mathrm{ng} / 1$ leptin in combination with AG490 (10 nM; Selleck Chemicals, Houston, TX, USA) were added. At various time points following this (12, 24, 48 or 72 h), $10 \%$ Cell Counting kit (CCK)-8 medium (Beyotime Institute of Biotechnology) diluted in serum-free DMEM was added to each well for $1 \mathrm{~h}$. The absorbance of each sample was measured at a wavelength of $450 \mathrm{~nm}$ using a Labsystems MK3 microplate reader (Thermo Fisher Scientific Inc., Waltham, MA, USA) to detect cell viability, according to the manufacturer's protocols. Cells not treated with leptin or AG490 served as the control group, and all experiments were performed in triplicate.
Apoptosis assay. Cells were washed with pre-warmed PBS, trypsinized and suspended in binding buffer (BD Biosciences, Franklin Lakes, NJ, USA) and the density was adjusted to $5-10 \times 10^{5} / \mathrm{ml}$. Apoptosis was measured by staining with Annexin V-fluorescein isothiocyanate (FITC) and propidium iodide $(\mathrm{PI})$. The cell suspension $(195 \mu \mathrm{l})$ was added to $5 \mu \mathrm{l}$ Annexin V-FITC and $10 \mu \mathrm{l}$ PI (BD Biosciences). Stained cells were analyzed by fluorescence-activated cell-sorting (FACS) (FACSCalibur, BD Biosciences). The apoptotic cells were reported as the percentage of cells that were Annexin-positive and PI-negative.

Measurement of intracellular ROS levels. The levels of intracellular ROS were measured using a ROS assay kit (Beyotime Institute of Biotechnology), performed according to the manufacturer's protocol. Treated chondrocytes were washed three times with pre-warmed PBS and harvested for staining. Dihydroethidium $(50 \mu \mathrm{M})$ was added to the cells and incubated at room temperature for $30 \mathrm{~min}$ prior to analysis using the FACSCalibur flow cytometer. The fluorescence intensity was monitored at an excitation wavelength of $488 \mathrm{~nm}$ and an emission wavelength of $605 \mathrm{~nm}$.

Western blot analysis. Chondrocytes were washed with PBS several times then added to lysis buffer (Beijing Solarbio Science \& Technology Co., Ltd., Beijing, China) with proteinase and phosphatase inhibitors (Sigma-Aldrich). The concentration of protein was quantified using the bicinchoninic acid method (Thermo Fisher Scientific, Inc.). A $35 \mu \mathrm{g}$ protein sample from each group was separated by $10 \%$ sodium dodecyl sulfate-polyacrylamide gel electrophoresis, prior to transfer to polyvinylidene fluoride membranes (Merck Millipore, Darmstadt, Germany). The blots were blocked with 5\% non-fat milk and incubated overnight with primary antibodies at $4^{\circ} \mathrm{C}$, as follows: Rabbit polyclonal anti-rat MMP-13 (1:2,500; cat. no. ab39012; Abcam); rabbit polyclonal anti-rat Beclin-1 (1:800; cat. no. ab62557; Abcam), rabbit polyclonal anti-rat B-cell lymphoma 2 (Bcl-2)-associated X protein (Bax; 1:100; cat. no. sc-493; Santa Cruz Biotechnology, Inc., Dallas, TX, USA); rabbit polyclonal anti-rat Bcl-2 (1:150; cat. no. sc-492; Santa Cruz Biotechnology, Inc.); rabbit polyclonal anti-microtubule-associated protein 1A/1B-light chain 3 (LC3) (1:200; cat. no. ab58610; Abcam); rabbit monoclonal anti-rat phosphorylated (p)-JAK2 (1:1,000; cat. no. 3776; Cell Signaling Technology, Inc., Danvers, MA, USA); rabbit monoclonal anti-rat JAK2 (1:1,000; cat. no. 3230; Cell Signaling Technology, Inc.); rabbit monoclonal anti-rat p-STAT3 (1:1,000; cat. no. ab76315; Abcam); mouse monoclonal anti-rat STAT3 (1:1,000; ab119352; Abcam); and rabbit monoclonal anti-rat glyceraldehyde 3-phosphate dehydrogenase (GAPDH; 1:1,500; cat. no. 5174; Cell Signaling Technology, Inc.). GAPDH served to normalize protein loading. Following three washes with Tris-buffered saline with $0.1 \%$ Tween 20 , the blots were incubated for $1 \mathrm{~h}$ at room temperature with horseradish peroxidase-conjugated goat anti-mouse (1:1,000; cat. no. A0216; Beyotime Institute of Biotechnology) or goat anti-rabbit (1:1,000; cat. no. A0208; Beyotime Institute of Biotechnology) secondary antibodies. The blots were washed again and the signals were analyzed using enhanced chemiluminescence (EMD Millipore, Billerica, MA, USA). Intensities were 

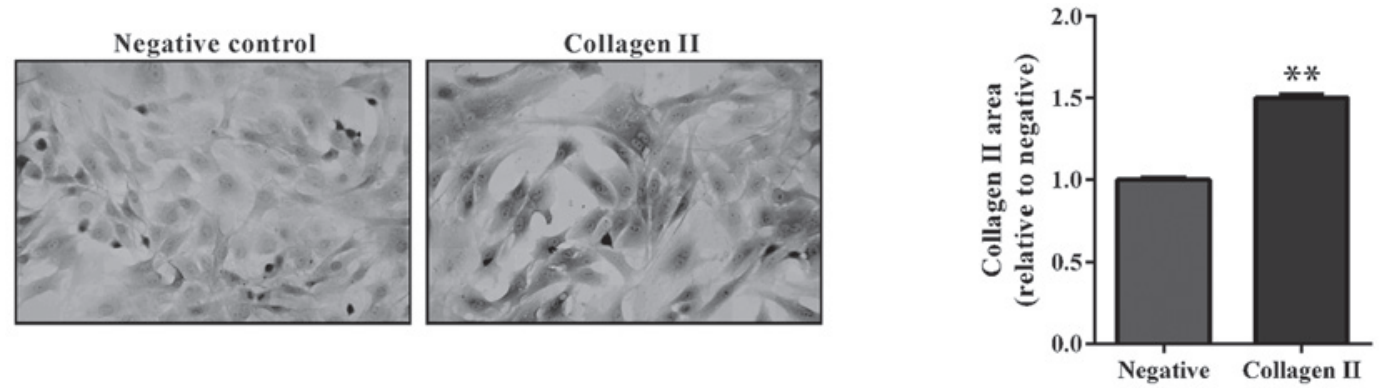

Figure 1. Identification of primary cultured chondrocytes. Freshly cultured chondrocytes from the control group (which did not undergo anterior cruciate ligament transection) were stained with collagen II as an indication of functional chondrocytes. The left image is of cells stained with immunoglobulin G as a negative control for the collagen II antibody. Areas of interest were selected at random and a positive area was calculated and analyzed. Data are presented as the mean \pm standard deviation. ${ }^{* *} \mathrm{P}<0.01$ vs. the negative control. Magnification, $\mathrm{x} 200$.
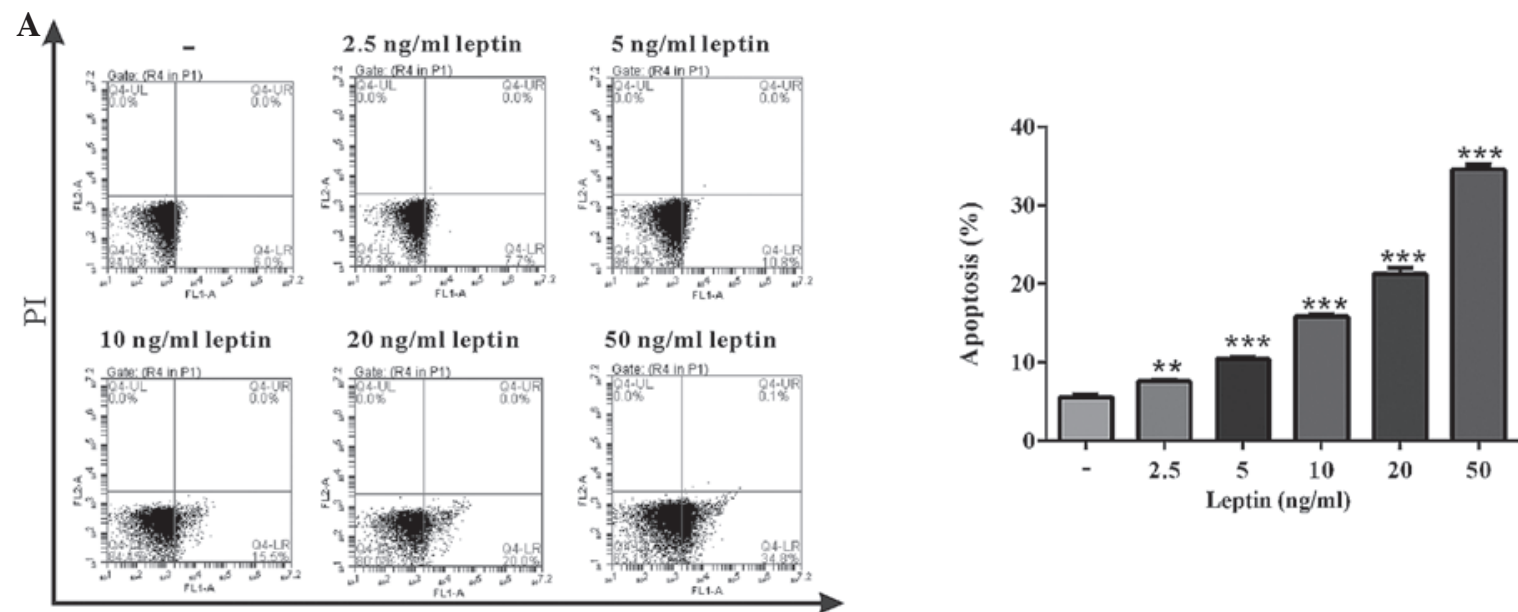

Annexin V
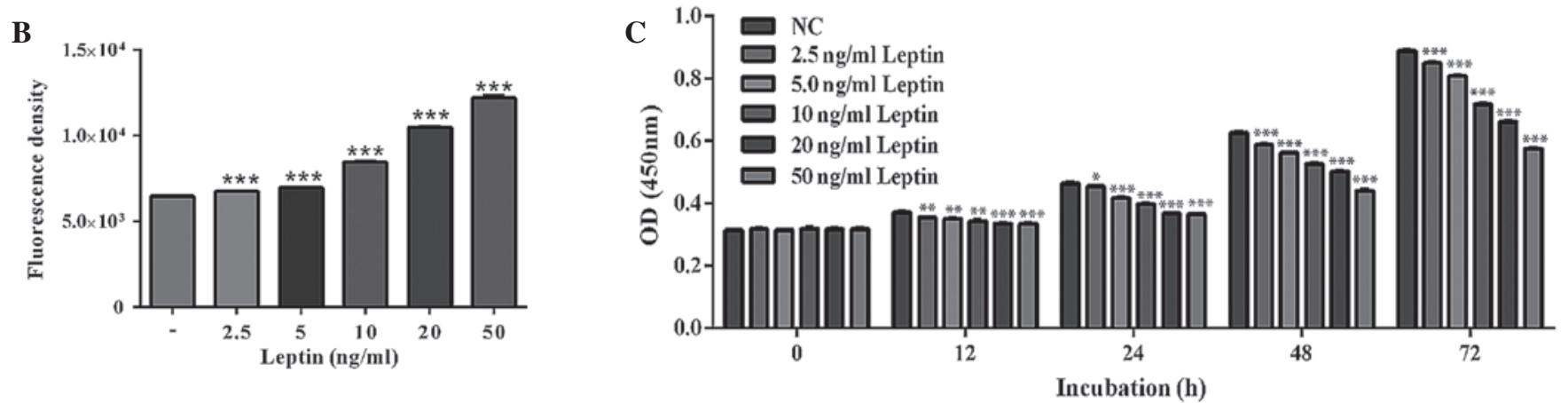

Figure 2. Leptin damaged chondrocyte viability, and induced apoptosis and ROS production. (A) Percentage of apoptotic chondrocytes, as measured by Annexin V-fluorescein isothiocyanate and PI staining. IV gated cells were indicators of apoptosis. (B) Detection of intracellular ROS was conducted using the probe DHE. Treated chondrocytes were stained with DHE followed by analysis using a fluorescence-activated cell sorting machine. (C) Chondrocyte viability was analyzed by Cell Counting kit-8 assay. A dose- and time-dependent effect of leptin on the viability of chondrocytes was observed. Data are presented as the mean \pm standard deviation. ${ }^{*} \mathrm{P}<0.05 ;{ }^{* *} \mathrm{P}<0.01 ;{ }^{* * *} \mathrm{P}<0.001$ vs. the control group. ROS, reactive oxygen species; DHE, dihydroethidium; OD, optical density; $\mathrm{PI}$, propidium iodide; $\mathrm{NC}$, negative control.

reported as the relative pixels normalized to those of GAPDH and measured using ImageJ software.

Statistical analysis. Statistical analysis was performed with one-way analysis of variance using GraphPad Prism version 6.0 software (GraphPad Software, Inc., La Jolla, CA, USA). Data were expressed as the mean \pm standard deviation of triplicate samples. All results were observed in at least three repeated experiments. $\mathrm{P}<0.05$ was considered to indicate a statistically significant difference.

\section{Results}

Leptin induces chondrocyte apoptosis and decreases cell viability. In order to investigate the function of leptin in $\mathrm{OA}$, an in vitro chondrocyte model was established from rat knee joints following ACLT surgery. Collagen II is abundantly expressed in functional chondrocytes, and in joint diseases characterized by low-grade inflammation and severe mechanical stress, including OA. Immunocytochemistry demonstrated that collagen II was expressed in primary 
A

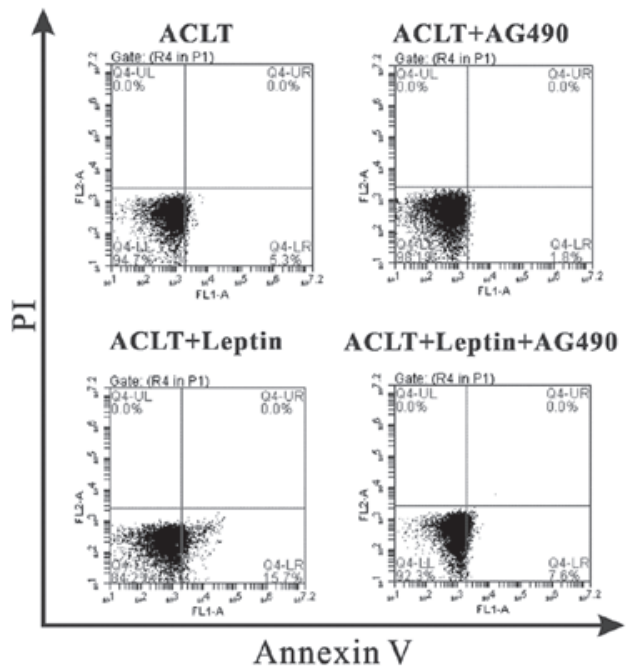

B

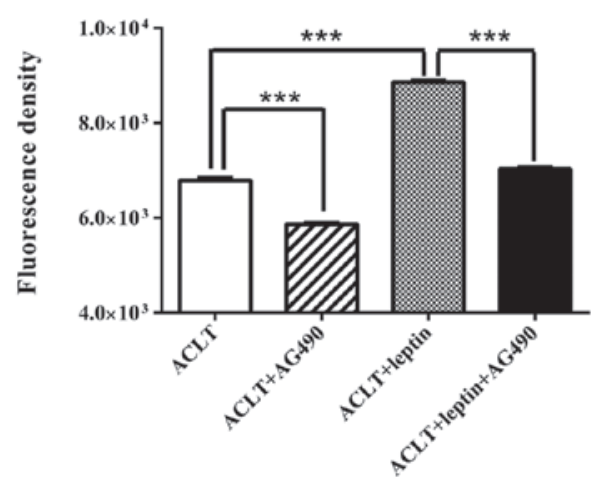

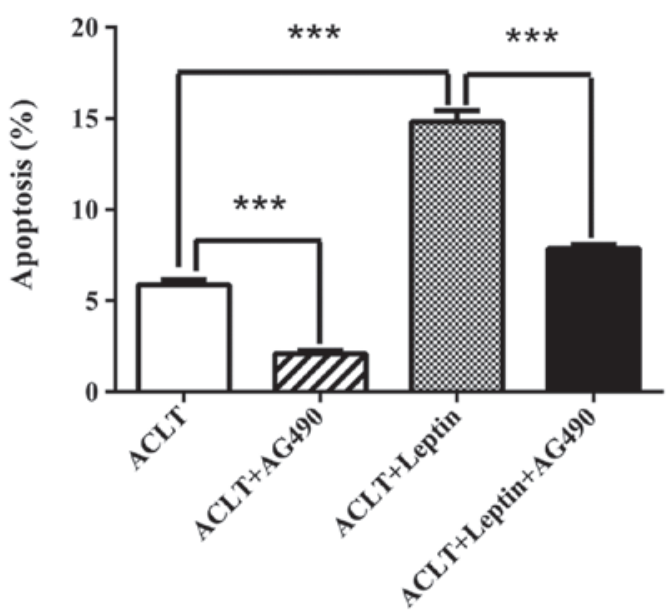

C

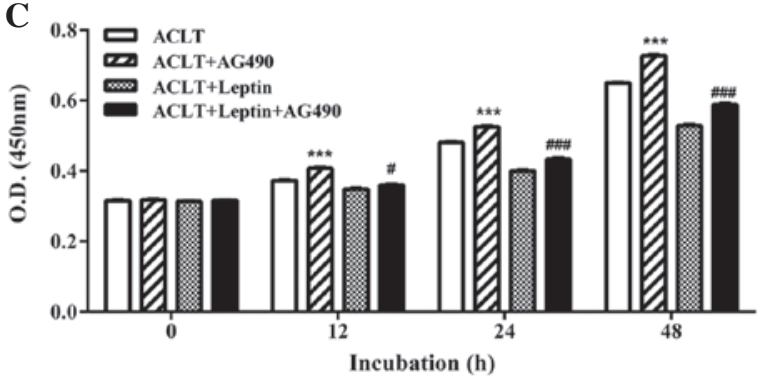

Figure 3. AG490 reduced leptin-induced chondrocyte apoptosis or ROS production, and restored viability. (A) Following a 48 h incubation, AG490 suppressed leptin-induced chondrocyte apoptosis. IV gated cells indicated apoptosis. ${ }^{* * * *} \mathrm{P}<0.001$. (B) Detection of intracellular ROS was conducted using the DHE probe. Treated chondrocytes were stained with DHE followed by analysis using a fluorescence-activated cell sorting machine. ${ }^{* * *} \mathrm{P}<0.001$. (C) Viability of the chondrocytes was tested using Cell Counting kit- 8 . A dose- and time-dependent effect of leptin on the viability of chondrocytes was observed. Data are presented as the mean \pm standard deviation. ${ }^{* * *} \mathrm{P}<0.001$, ACLT + AG490 group vs. ACLT group; ${ }^{\#} \mathrm{P}<0.05,{ }^{\# \#} \mathrm{P}<0.001$, ACLT + leptin + AG490 group vs. ACLT + leptin group. ROS, reactive oxygen species; DHE, dihydroethidium; ACLT, anterior cruciate ligament transection; PI, propidium iodide; OD, optical density.

cultured chondrocytes (Fig. 1), which was indicative of a chondrocyte model.

Following treatment with leptin for $48 \mathrm{~h}$, chondrocytes were stained with Annexin V for further analysis (Fig. 2). The FACS results indicated that chondrocytes underwent marked apoptosis following administration of leptin in a dose-dependent manner (from 7.60 to $34.60 \%$ ), as compared with the control group (5.63\%) (Fig. 2A). Leptin is a proinflammatory adipokine that is able to generate ROS production in a number of disease models (14). In order to address this in the current model, ROS production was measured in injured chondrocytes. Treatment with various quantities of leptin for $48 \mathrm{~h}$ markedly induced the generation of ROS by mitochondria (Fig. 2B). Furthermore, the CCK- 8 assay indicated that the viability of chondrocytes was reduced in an increasingly dose-dependent manner over time in response to leptin treatment (Fig. 2C).

Inhibition of JAK2-STAT3 signaling ameliorates leptin-induced chondrocyte apoptosis and rescues reduced cell viability. Leptin binding triggers the leptin receptor to recruit cytoplasmic kinases, including JAK2, to initiate leptin signaling (15). Previous studies have indicated that selective inhibition of JAK signaling has a beneficial effect in modulating leptin-associated diseases $(16,17)$. Following the administration of the JAK2 inhibitor, AG490, injured chondrocytes were rescued from leptin-induced apoptosis. As presented in Fig. 3A, incubation with AG490 alone for $48 \mathrm{~h}$ decreased the basal level of apoptosis in ACLT chondrocytes (from 5.87 to 2.10\%). Co-incubation with leptin and AG490 significantly reduced leptin-induced apoptosis almost to basal levels (from 14.83 to $7.87 \%$ ). In addition, AG490 reduced ROS production induced by leptin (Fig. 3B). The production of ROS in the AG490 + leptin group was decreased to almost the same level as the ACLT group.

Furthermore, the CCK- 8 assay demonstrated that AG490 restored chondrocyte viability in leptin-treated and -untreated cells. After a $48 \mathrm{~h}$ incubation, AG490 increased chondrocyte viability by 11.84 and $11.32 \%$ in leptin-untreated and -treated cells, respectively (Fig. 3C).

These results suggest that as a proinflammatory adipokine, leptin may result in damage to chondrocytes, and JAK2 inhibition may protect chondrocytes via clearance of ROS and inhibition of apoptosis, thereby restoring cell viability.

Inhibition of the JAK2-STAT3 signaling pathway alters protein expression associated with apoptosis and autophagy 
A

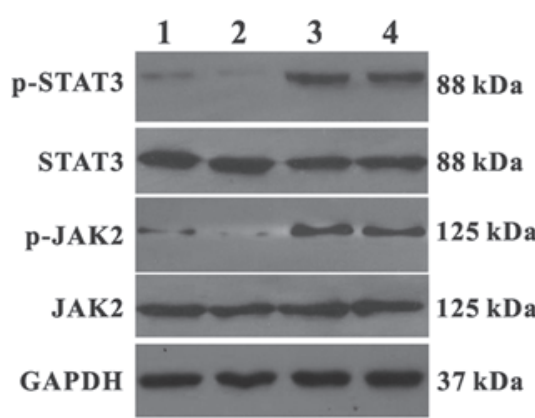

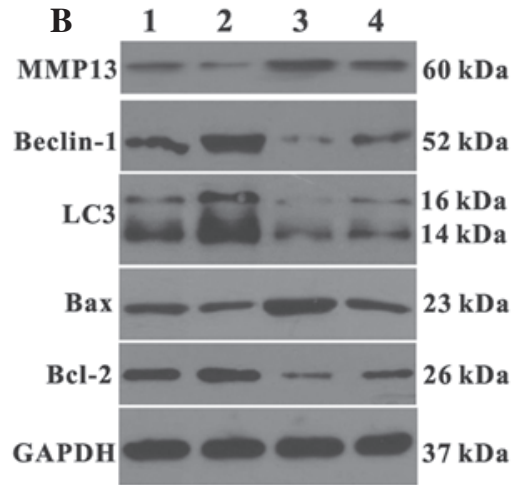

D

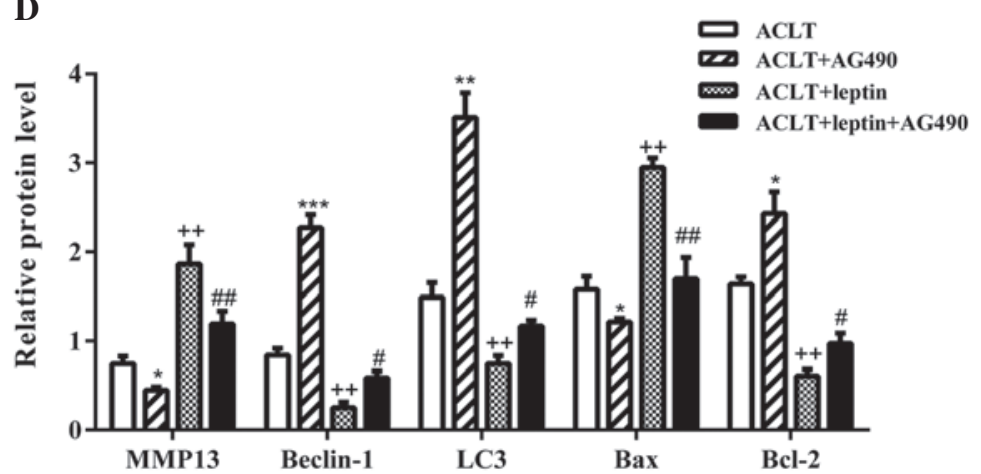

Figure 4. Western blot analysis of JAK-STAT signaling activity and apoptosis/autophagy-associated protein expression. (A) A $4 \mathrm{~h}$ treatment with AG490 or leptin + AG490 inhibited phosphorylation of STAT3 and JAK2. GAPDH served as an internal loading control. (B) Following incubation for $24 \mathrm{~h}$, a number of autophagy key regulators, including Beclin-1 and LC3, and apoptotic markers were detected. (C and D) Quantification of the western blotting is presented. Lane 1, ACLT group; lane 2, ACLT + AG490; lane 3, ACLT + leptin; lane 4, ACLT + leptin + AG490. Molecular weight for each protein is labeled. Data are presented as the mean \pm standard deviation. ${ }^{*} \mathrm{P}<0.05$, AG490 group vs. ACLT group; ${ }^{+} \mathrm{P}<0.05,{ }^{++} \mathrm{P}<0.01$, ACLT + leptin group vs. ACLT group; ${ }^{\#} \mathrm{P}<0.05$, ${ }^{\# "} \mathrm{P}<0.01$, ACLT + leptin + AG490 group vs. ACLT + leptin group. JAK, Janus kinase; STAT, signal transducer and activator of transcription; LC3, microtubule-associated protein 1A/1B-light chain 3; ACLT, anterior cruciate ligament transection; MMP-13, matrix metalloproteinase-13; Bcl-2, B-cell lymphoma 2; Bax, Bcl-2-associated X protein; GAPDH, glyceraldehyde 3-phosphate dehydrogenase.

in chondrocytes following leptin-induced damage. The present study demonstrated that selective inhibition of the JAK2-STAT3 signaling pathway by AG490 may protect chondrocytes from leptin-induced apoptosis. Therefore, certain proteins associated with apoptosis and the JAK2-STAT3 signaling pathway were detected (Fig. 4). Upon environmental stimulus, leptin binds to its receptor and recruits cytoplasmic JAK2 to initiate leptin signaling. Activation of JAK2 by auto-phosphorylation initiates recruitment and phosphorylation of STAT3 for additional effects (18). As shown in Fig. 4A and C, incubation with leptin for $3 \mathrm{~h}$ notably induced phosphorylation of JAK2 and STAT3, and administration of $10 \mathrm{nM}$ of AG490 significantly inhibited activities of JAK2/STAT3 in the leptin-treated and -untreated groups. Leptin has been reported to increase the production of MMPs, which belong to a large family of proteases that degrade the extracellular matrix and directly damage cartilage (10). In addition to reducing apoptosis, the expression levels of MMP-13 were reduced following treatment with AG490 for $24 \mathrm{~h}$, indicating improved chondrocyte function (Fig. 4B and D).

The $\mathrm{Bax} / \mathrm{Bcl}-2$ family is essential for the regulation of apoptosis, and an increased ratio of $\mathrm{Bax} / \mathrm{Bcl}-2$ results in caspase activation and downstream apoptosis (19). Leptin increased Bax expression and suppressed Bcl-2 expression; however, this was reversed by AG490 (Fig. 4B).

Unlike apoptosis, autophagy in normal adult articular cartilage is an important mechanism for cellular homeostasis, and may be complicated depending on the stage of development (20). Under the majority of circumstances, autophagy promotes cell survival by adapting cells to stress conditions. In an OA model, autophagy was reduced alongside a decrease in the key regulators, including autophagy protein (ATG)5, LC3 and Beclin-1 (21). In the present study, detection of Beclin-1 and LC3 expression was conducted in ACLT chondrocytes; leptin reduced the expression levels of Beclin-1 and LC3, which were restored by AG490 (Fig. 4B and D).

\section{Discussion}

Obesity is a global public health problem, which is identified as a metabolic low-grade inflammatory condition. Obesity is characterized by articular cartilage degradation, subchondral bone sclerosis, osteophyte formation and synovial inflammation (2). OA is strongly associated with obesity, beyond the burden of excess body weight on weight-bearing joints (22), for example, a previous study reported a two-fold increase in hand OA risk in obese individuals (3). Increasing evidence has indicated that metabolic factors released by WAT may be responsible for the high prevalence of OA among obese people (4). Among the adipose-derived molecules, referred to as adipokines, leptin has been most investigated. Leptin is a proinflammatory adipokine, the expression levels of which are positively correlated with body mass index (BMI) (23). Obese individuals generally exhibit a high level of circulating 
leptin, which is associated with higher prevalence of knee OA, following age, ethnicity and BMI adjustments $(5,24)$. In addition, leptin is observed in synovial fluid at three times higher concentrations than in non-obese individuals (25). This leptin is released by local chondrocytes (26), thus indicating that local and systemic leptin may have distinct functions in bone metabolism.

The expression of collagen II is used as a marker for the healthy chondrocyte phenotype, and is also observed in primary cultured rat chondrocytes (27). The expression of leptin receptors in human chondrocytes was originally detected by Figenschau et al (6), which indicated that leptin directly participated in joint damage by initiating iNOS production. In addition, this injury required other proinflammatory cytokines, including interferon (IFN)- $\gamma$ or interleukin (IL)-1 $(9,11)$. The in vivo ACLT model established in the present study provided an ideal inflammatory environment for elucidating the function of leptin in vitro without administration of more proinflammatory cytokines.

A previous study has reported that leptin triggers the expression of iNOS, alone or in combination with IL-1 $\beta$, to potentially induce apoptosis in human or rodent chondrocytes (7). In the present study, leptin was demonstrated to induce apoptosis and reduce cell viability in a dose- and time-dependent manner. Furthermore, damaged chondrocytes themselves, which were used in the current system, may release inflammatory cytokines that associate with leptin to trigger this deterioration process. However, the specific effective cytokines and detailed underlying mechanisms remain to be elucidated and require further study in the future.

During cellular stress, mitochondrial membrane potential may be altered by dysfunction of intracellular ionic charge, leading to the generation of ROS, which is a key indicator of cell death or injury. It has previously been demonstrated that oxidative damage as a result of ROS contributes to chondrocyte apoptosis (28), and treatment of human chondrocytes with an anti-oxidative agent may result in a prolonged replicative lifespan (29). However, to the best of our knowledge, no previous studies have addressed the possible involvement of leptin in ROS production, and the present study reports for the first time that leptin contributes to ROS-induced oxidative stress in chondrocytes.

Leptin exerts a proinflammatory effect via JAK2, PI3K and MAPKs in chondrocytes, and selective blocking of individual intracellular signaling pathways may exert beneficial effects. Upon leptin binding, the leptin receptor recruits cytoplasmic kinases, such as JAK2, and forms a receptor homodimer to facilitate auto-phosphorylation and activation of JAK 2 in order to initiate leptin signaling. Furthermore, activation of JAK2 initiates recruitment of STAT members, including STAT3, for subsequent signal transduction (15). Using the specific JAK2 inhibitor, AG490, the JAK2 signaling pathway was revealed to be associated with the effects of leptin on apoptosis of chondrocytes. Otero et al $(9,11)$ demonstrated that inhibition of the JAK2-STAT3 signaling pathway with AG490 may result in the suppression of leptin-induced iNOS expression with IL-1 or IFN- $\gamma$ in human chondrocytes. In the present study, in addition to protection from apoptosis, AG490 increased cell viability and reduced the production of ROS, suggesting its additional protective influence on cell viability. Key factors in associated signaling pathways also indicated the same outcomes; the phosphorylation levels of JAK2 and STAT3 were reduced in the AG490 group with or without leptin stimulation. Consistent with the results from the ROS detection and apoptosis experiments, the ratio of $\mathrm{Bcl}-2 / \mathrm{Bax}$ was upregulated, indicating a reduction in apoptosis.

The function of autophagy in chondrocytes is complex and involves numerous mediators produced by chondrocytes. Autophagy maintains cellular homeostasis in normal adult articular cartilage and chondrocytes. Chondrocytes in the superficial zone exhibit a marked expression of autophagy proteins, Beclin-1, ATG5 and LC3 (30), and during the damaging process of OA, decreases in Beclin-1 and LC3 are accompanied by increased apoptosis (30). In the present study, AG490 reduced apoptosis in chondrocytes and restored Beclin-1 and LC3 expression, which may be beneficial for improving normal cellular functions.

In conclusion, as a proinflammatory adipokine, leptin markedly reduced chondrocyte function, and induced apoptosis, in an in vitro OA model. The JAK2-STAT3 signaling pathway was considered the underlying molecular mechanism by which leptin induces apoptosis, and blocking this signal transduction in affected joints may be considered a promising therapeutic target during OA therapy.

\section{Acknowledgements}

The present study was supported by the Natural Science Foundation of China (grant no. 81372001) and the Science and Technology Commission of Shanghai Municipality Fund (grant no. 13ZR1427000).

\section{References}

1. Felson DT: An update on the pathogenesis and epidemiology of osteoarthritis. Radiol Clin North Am 42: 1-9, 2004.

2. Gregor MF and Hotamisligil GS: Inflammatory mechanisms in obesity. Annu Rev Immunol 29: 415-445, 2011.

3. Yusuf E, Nelissen RG, Ioan-Facsinay A, Stojanovic-Susulic V, DeGroot J, van Osch G, Middeldorp S, Huizinga TW and Kloppenburg M: Association between weight or body mass index and hand osteoarthritis: A systematic review. Ann Rheum Dis 69: 761-765, 2010

4. Pottie P, Presle N, Terlain B, Netter P, Mainard D and Berenbaum F: Obesity and osteoarthritis: More complex than predicted! Ann Rheum Dis 65: 1403-1405, 2006

5. Dumond H, Presle N, Terlain B, Mainard D, Loeuille D, Netter P and Pottie P: Evidence for a key role of leptin in osteoarthritis. Arthritis Rheum 48: 3118-3129, 2003.

6. Figenschau Y, Knutsen G, Shahazeydi S, Johansen O and Sveinbjörnsson B: Human articular chondrocytes express functional leptin receptors. Biochem Biophys Res Commun 287: 190-197, 2001.

7. Vuolteenaho K, Koskinen A, Kukkonen M, Nieminen R, Päivärinta U, Moilanen T and Moilanen E: Leptin enhances synthesis of proinflammatory mediators in human osteoarthritic cartilage-mediator role of NO in leptin-induced PGE2, IL-6 and IL-8 production. Mediators Inflamm 2009: 345838, 2009.

8. Li D, Xie G and Wang W: Reactive oxygen species: The 2-edged sword of osteoarthritis. Am J Med Sci 344: 486-490, 2012

9. Otero M, Lago R, Lago F, Reino JJ and Gualillo O: Signalling pathway involved in nitric oxide synthase type II activation in chondrocytes: Synergistic effect of leptin with interleukin-1. Arthritis Res Ther 7: R581-R591, 2005.

10. Koskinen A, Vuolteenaho K, Nieminen R, Moilanen T and Moilanen E: Leptin enhances MMP-1, MMP-3 and MMP-13 production in human osteoarthritic cartilage and correlates with MMP-1 and MMP-3 in synovial fluid from OA patients. Clin Exp Rheumatol 29: 57-64, 2011. 
11. Otero M, Gomez Reino JJ and Gualillo O: Synergistic induction of nitric oxide synthase type II: In vitro effect of leptin and interferon-gamma in human chondrocytes and ATDC5 chondrogenic cells. Arthritis Rheum 48: 404-409, 2003.

12. Pickarski M, Hayami T, Zhuo Y and Duong LT: Molecular changes in articular cartilage and subchondral bone in the rat anterior cruciate ligament transection and meniscectomized models of osteoarthritis. BMC Musculoskelet Disord 12: 197, 2011.

13. Tew SR, Murdoch AD, Rauchenberg RP and Hardingham TE: Cellular methods in cartilage research: Primary human chondrocytes in culture and chondrogenesis in human bone marrow stem cells. Methods 45: 2-9, 2008

14. Hamada Y, Fujii $\mathrm{H}$ and Fukagawa M: Role of oxidative stress in diabetic bone disorder. Bone 45 (Suppl 1): S35-S38, 2009.

15. Ghilardi N and Skoda RC: The leptin receptor activates janus kinase 2 and signals for proliferation in a factor-dependent cell line. Mol Endocrinol 11: 393-399, 1997.

16. Ohba S, Lanigan TM and Roessler BJ: Leptin receptor JAK2/ STAT3 signaling modulates expression of Frizzled receptors in articular chondrocytes. Osteoarthritis Cartilage 18: 1620-1629, 2010.

17. Mutabaruka MS, Aoulad Aissa M, Delalandre A, Lavigne M and Lajeunesse D: Local leptin production in osteoarthritis subchondral osteoblasts may be responsible for their abnormal phenotypic expression. Arthritis Res Ther 12: R20, 2010.

18. Bahrenberg G, Behrmann I, Barthel A, Hekerman P, Heinrich PC, Joost HG and Becker W: Identification of the critical sequence elements in the cytoplasmic domain of leptin receptor isoforms required for Janus kinase/signal transducer and activator of transcription activation by receptor heterodimers. Mol Endocrinol 16: 859-872, 2002.

19. Adams JM and Cory S: Life-or-death decisions by the Bcl-2 protein family. Trends Biochem Sci 26: 61-66, 2001.
20. Lotz MK and Caramés B: Autophagy and cartilage homeostasis mechanisms in joint health, aging and osteoarthritis. Nat Rev Rheumatol 7: 579-587, 2011.

21. Shapiro IM, Layfield R, Lotz M, Settembre C and Whitehouse C: Boning up on autophagy: The role of autophagy in skeletal biology. Autophagy 10: 7-19, 2014.

22. Aspden RM: Obesity punches above its weight in osteoarthritis Nat Rev Rheumatol 7: 65-68, 2011.

23. Friedman JM and Halaas JL: Leptin and the regulation of body weight in mammals. Nature 395: 763-770, 1998.

24. Gualillo O, Eiras S, Lago F, Diéguez C and Casanueva FF: Elevated serum leptin concentrations induced by experimental acute inflammation. Life Sci 67: 2433-2441, 2000.

25. Presle N, Pottie P, Dumond H, Guillaume C, Lapicque F, Pallu S, Mainard D, Netter P and Terlain B: Differential distribution of adipokines between serum and synovial fluid in patients with osteoarthritis. Contribution of joint tissues to their articular production. Osteoarthritis Cartilage 14: 690-695, 2006.

26. Distel E, Cadoudal T, Durant S, Poignard A, Chevalier X and Benelli C: The infrapatellar fat pad in knee osteoarthritis: An important source of interleukin-6 and its soluble receptor. Arthritis Rheum 60: 3374-3377, 2009.

27. Goldring MB, Tsuchimochi K and Ijiri K: The control of chondrogenesis. J Cell Biochem 97: 33-44, 2006.

28. Hashimoto S, Takahashi K, Amiel D, Coutts RD and Lotz M Chondrocyte apoptosis and nitric oxide production during experimentally induced osteoarthritis. Arthritis Rheum 41: $1266-1274,1998$

29. Henrotin Y and Kurz B: Antioxidant to treat osteoarthritis: Dream or reality? Curr Drug Targets 8: 347-357, 2007.

30. Almonte-Becerril M, Navarro-Garcia F, Gonzalez-Robles A, Vega-Lopez MA, Lavalle C and Kouri JB: Cell death of chondrocytes is a combination between apoptosis and autophagy during the pathogenesis of Osteoarthritis within an experimental model. Apoptosis 15: 631-638, 2010 